\title{
Genetic testing for lymphedema-distichiasis syndrome
}

\author{
Yeltay Rakhmanov ${ }^{1}$, Paolo Enrico Maltese ${ }^{1 \star}$, Stefano Paolacci ${ }^{2}$, Carla Marinelli ${ }^{1}$ and Matteo Bertelli ${ }^{1,2}$
}

\begin{abstract}
We studied the scientific literature and disease guidelines to summarize the clinical utility of genetic testing for lymphedema distichiasis (LD) syndrome. LD is inherited in an autosomal dominant manner, and has unknown prevalence. It is caused by variations in the FOXC2 gene. Clinical diagnosis involves clinical examination, targeted at identifying primary lymphedema (chronic swelling of the extremities) and distichiasis (double row of eyelashes). The genetic test is useful for confirming diagnosis, as well as for differential diagnosis, couple risk assessment and access to clinical trials.
\end{abstract}

Keywords: Lymphedema-distichiasis syndrome, FOXC2, EBTNA UTILITY GENE TEST

'MAGI Euregio, Bolzano, Italy

${ }^{2}$ MAGI's Lab, Rovereto, Italy

*Corresponding author: P. E. Maltese E-mail: paolo.maltese@assomagi.org

DOI: $10.2478 /$ ebtj-2018-0026 (c) 2018 Authors. This work was licensed under the Creative Commons AttributionNonCommercial-NoDerivs 3.0 License.

\section{Lymphedema-distichiasis syndrome}

(Other synonyms: Distichiasis-lymphedema syndrome; lymphedema with distichiasis)

\section{General information about the disease}

Lymphedema-distichiasis (LD, OMIM disease 153400) syndrome is a rare genetic disorder that affects the lymphatic system. It combines lymphedema of the lower extremities and double rows of eyelashes (aberrant eyelashes ranging from a full set of extra eyelashes to a single hair) (1). Affected individuals may also have renal abnormalities (nephritis, duplex kidney, recurrent infections, chronic sclerosing glomerulopathy), diabetes mellitus (2), varicose veins, cleft palate, extradural cysts, congenital heart defects (tetralogy of Fallot, ventricular septal defects), ocular findings, such as photophobia, exotropia, droopy eyelids (ptosis), ectropion and cataracts $(3,4)$.

The estimated prevalence of LD is currently unknown.

The clinical diagnosis of LD is based on: primary lymphedema (chronic swelling of the extremities caused by intrinsic dysfunction of the lymphatic vessels); distichiasis (aberrant and/or extra row of eyelashes originating from the tarsal gland) (1).

Approximately $80 \%$ of individuals with lymphedema-distichiasis syndrome have lymphedema by early adulthood (age 30 years), although a few individuals may develop lymphedema later. Approximately $94 \%$ of affected individuals also have distichiasis (5).

Differential diagnosis is necessary with respect to other diseases that manifest lymphedema or distichiasis, including Milroy disease, Meige disease microcephaly with/without chorioretinopathy, lymphedema, or mental retardation, Emberger syndrome, hypotrichosis lymphedema telangiectasia syndrome, yellow nail syndrome, blepharocheilodontic syndrome, isolated distichiasis, and trichiasis (1).

LD is inherited in an autosomal dominant manner, although approximately one quarter of cases are the result of de novo mutations. Fang et al. identified mutations in the FOXC2 (Forkhead Box C2, OMIM gene 602402) gene as a cause of LD Syndrome. The single exon $1.5 \mathrm{~kb}$ FOXC2 gene is a member of the 'forkhead' (or winged helix) gene family, originally identified in Drosophila, which encodes transcription factors with a 
conserved 100-amino acid DNA binding motif. About 60 different causative FOXC2 mutations have been reported (2). The specific function of FOXC2 has not yet been determined; however, it may play a role in mesenchymal and adipose tissues, and heart development (6), and has been found to play a role in the Hedgehog (Hh) signaling pathway (7).

Pathogenic variants in FOXC2 are identified in 95\% of cases with LD (1). Since 5\% of patients with LD does not carry pathogenic variants in FOXC2, genetic heterogeneity can be hypothesized.

Pathogenic variants are either gain-of-function or loss-offunction and may include missense, nonsense, small insertions and deletions, small indels and gross deletions or duplications.

\section{Aims of the test}

- To determine the gene defect responsible for the disease;

- To confirm clinical diagnosis;

- To assess the recurrence risk and perform genetic counselling for at-risk/affected individuals.

\section{Test characteristics}

\section{Specialist centers/ Published Guidelines}

The test is listed in the Orphanet database and is offered by 47 accredited medical genetic laboratories in the EU, and in the GTR database, offered by 14 accredited medical genetic laboratories in the US.

Guidelines for clinical use of the test are described in Genetics Home Reference (ghr.nlm.nih.gov) and Gene Reviews (1).

\section{Test strategy}

Since it is single gene disorder, we advise Sanger sequencing for the detection of nucleotide variations in coding exons and flanking introns in the FOXC2 gene. Sanger sequencing is also used for family segregation studies.

Multiplex Ligation Probe Amplification (MLPA) is used to detect duplications and deletions in FOXC2.

To perform molecular diagnosis, a single sample of biological material is normally sufficient. This may be $1 \mathrm{ml}$ peripheral blood in a sterile tube with $0.5 \mathrm{ml} \mathrm{K}$ EDTA or $1 \mathrm{ml}$ saliva in a sterile tube with $0.5 \mathrm{ml}$ ethanol $95 \%$. Sampling rarely has to be repeated. Gene-disease associations and the interpretation of genetic variants are rapidly developing fields. It is therefore possible that the genes mentioned in this note may change as new scientific data is acquired. It is also possible that genetic variants today defined as of "unknown or uncertain significance" may acquire clinical importance.

\section{Genetic test results}

\section{Positive}

Identification of pathogenic variants in the FOXC2 confirms the clinical diagnosis and is an indication for family studies.

A pathogenic variant is known to be causative for a given genetic disorder based on previous reports, or predicted to be causative based on loss of protein function or expected significant damage to proteins or protein/protein interactions. In this way it is possible to obtain a molecular diagnosis in new/other subjects, establish the risk of recurrence in family members and plan preventive and/or therapeutic measures.

\section{Inconclusive}

Detection of a variant of unknown or uncertain significance (VUS): a new variation without any evident pathogenic significance or a known variation with insufficient evidence (or with conflicting evidence) to indicate it is likely benign or likely pathogenic for a given genetic disorder. In these cases, it is advisable to extend testing to the patient's relatives to assess variant segregation and clarify its contribution. In some cases, it could be necessary to perform further examinations/tests or to do a clinical reassessment of pathological signs.

\section{Negative}

The absence of variations in the genomic regions investigated does not exclude a clinical diagnosis but suggests the possibility of:

- alterations that cannot be identified by sequencing, such as large rearrangements that cause loss (deletion) or gain (duplication) of extended gene fragments;

- sequence variations in gene regions not investigated by this test, such as regulatory regions (5' and 3' UTR) and deep intronic regions;

- variations in other genes not investigated by the present test.

\section{Unexpected}

Unexpected results may emerge from the test, for example information regarding consanguinity, absence of family correlation or other genetically based diseases.

\section{Risk for progeny}

In autosomal dominant transmission, the probability that an affected carrier transmit the variant to his/her children is $50 \%$ in any pregnancy, irrespective of the sex of the child conceived.

\section{Limits of the test}

The test is limited by current scientific knowledge regarding the gene and disease.

Analytical sensitivity (proportion of positive tests when the genotype is truly present) and specificity (proportion of negative tests when the genotype is not present)

SANGER Analytical sensitivity $>99.99 \%$; Analytical specificity 99.99\%.

MLPA Analytical sensitivity $>99.99 \%$; Analytical specificity 99.99\%.

\section{Clinical sensitivity (proportion of positive tests} if the disease is present) and clinical specificity (proportion of negative tests if the disease is not present)

Clinical sensitivity: Variations in FOXC2 gene are identified in $95 \%$ of $\mathrm{LD}(1)$. 
Clinical specificity is estimated at approximately $99.99 \%$ (Author's laboratory data) (8).

\section{Prescription appropriateness}

The genetic test is appropriate when:

a) the patient meets the diagnostic criteria for LD;

b) the sensitivity of the test is greater than or equal to that of tests described in the literature.

\section{Clinical utility}

\section{Clinical management}

Confirmation of clinical diagnosis

Differential diagnosis

Yes

Couple risk assessment

Yes

Availability of clinical trials can be checked on-line at https://clinicaltrials.gov/

\section{References}

1. Mansour S, Brice GW, Jeffery S, Mortimer P. LymphedemaDistichiasis Syndrome. In: MP Adam, HH Ardinger, RA Pagon, SE Wallace, LJH Bean, et al., editors. GeneReviews(R). 1993. Seattle (WA).
2. Yildirim-Toruner C, Subramanian K, El Manjra L, Chen E, Goldstein $S$, Vitale E. A novel frameshift mutation of FOXC2 gene in a family with hereditary lymphedema-distichiasis syndrome associated with renal disease and diabetes mellitus. Am J Med Genet A 2004; 131(3): 281-86.

3. Fang J, Dagenais SL, Erickson RP, Arlt MF, Glynn MW, Gorski JL, Seaver LH, Glover TW. Mutations in FOXC2 (MFH-1), a forkhead family transcription factor, are responsible for the hereditary lymphedema-distichiasis syndrome. Am J Hum Genet 2000; 67(6): 1382-1388.

4. National Library of Medicine (US). Lymphedema-distichiasis syndrome. Bethesda (MD): The Library; Genetics Home Reference. 2014 (Internet).

5. Brice G, Mansour S, Bell R, Collin JR, Child AH, Brady AF, Sarfarazi M, Burnand KG, Jeffery S, Mortimer P, Murday VA. Analysis of the phenotypic abnormalities in lymphoedema-distichiasis syndrome in 74 patients with FOXC2 mutations or linkage to $16 q 24$. J Med Genet 2002; 39(7): 478-83.

6. http://www.genecards.org/cgi-bin/carddisppl?gene=FOXC2\& keywords=foxc2\#summaries

7. Zhang Y, Zhang Y. Forkhead box C2 promotes the invasion ability of human trophoblast cells through Hedgehog $(\mathrm{Hh})$ signaling pathway. Cell Biol Int. 2018; (Epub ahead of print).

8. Chen B, Gagnon M, Shahangian S, Anderson NL, Howerton DA, Boone JD; Centers for Disease Control and Prevention (CDC). Good Laboratory Practices for Molecular Genetic Testing for Heritable Diseases and Conditions. MMWR Recomm Rep. 2009; 58(RR-6):1-37; quiz CE-1-4. 